\title{
A deep level transient spectroscopy study of beryllium implanted $\boldsymbol{n}$-type $6 \mathrm{H}-\mathrm{SiC}$
}

\author{
X. D. Chen, S. Fung, ${ }^{\text {a) }}$ and C. D. Beling \\ Department of Physics, The University of Hong Kong, Hong Kong, People's Republic of China \\ M. Gong \\ Department of Physics, Sichuan University, Chengdu, Sichuan 610064, People's Republic of China
}

T. Henkel, H. Tanoue, and N. Kobayashi

Electrotechnical Laboratory, Tsukuba, Ibaraki 305-8568, Japan

(Received 16 February 2000; accepted for publication 23 May 2000)

\begin{abstract}
Beryllium implantation induced defects in $6 \mathrm{H}-\mathrm{SiC} p n$ junctions have been investigated by deep level transient spectroscopy. Five defect centers labeled BE1, BE2, BE3, BE4, and BE5 have been detected in the temperature range $100-450 \mathrm{~K}$. A comparative study has also been performed in low beryllium doped $n$-type $6 \mathrm{H}-\mathrm{SiC}$, which proved that the $\mathrm{BE} 1, \mathrm{BE} 2$, and $\mathrm{BE} 3$ centers are electron traps located at $0.34,0.44$, and $0.53 \mathrm{eV}$, respectively, below the conduction band edge. On the other hand, the BE4 and BE5 centers have been found to be hole traps which are situated at 0.64 and 0.73 $\mathrm{eV}$, respectively, above the valence band edge. Possible defect configurations associated with these deep levels are discussed. () 2000 American Institute of Physics. [S0021-8979(00)03817-2]
\end{abstract}

\section{INTRODUCTION}

Silicon carbide $(\mathrm{SiC})$ has long been recognized as a candidate material for high frequency, high temperature, and high power electron devices. The fabrication of electronic devices requires the modification of electronic properties by doping. Since the diffusion coefficients of the common dopants in $\mathrm{SiC}$ are low at temperatures where masking layers can be used, diffusion based techniques are not applicable for this purpose. ${ }^{1,2}$ Therefore, the development of the ion implantation process for $\mathrm{SiC}$ device technology is of importance. $\mathrm{SiC}$ implanted with gallium $(\mathrm{Ga})$, aluminum $(\mathrm{Al})$, and boron (B) were intensively studied in the past. ${ }^{3-9}$ However, the electrical activation of these dopants is limited due to high acceptor levels and low hole mobilities. ${ }^{5,9}$ Thus, alternative dopants with higher electrical activation are desirable.

Beryllium (Be), being a light element of group II-A with the same electronegativity as $\mathrm{Al}$ (1.5), is expected to induce less implantation damage when compared with $\mathrm{Al}$ or B. Further, Be can be implanted to deeper regions than B which might be useful for the generation of thick $p$-type layers in $\mathrm{SiC}$.

$\mathrm{Be}$ is known as a doubly charged acceptor in SiC. The acceptor levels, 0.42 and $0.60 \mathrm{eV}$ were determined at a first attempt by Hall measurements on $6 \mathrm{H}-\mathrm{SiC}$ bulk material. ${ }^{10}$ Recently, Be was successfully applied in the fabrication of SiC diodes. ${ }^{11}$ Current-voltage measurements on these $p^{+} n$ junctions provided an electronic level at $0.38 \mathrm{eV}$, which was attributed to the first (shallow) acceptor level. A better forward characteristic was obtained in comparison to $p n$ junctions produced by $\mathrm{B}$ implantation. On the other hand, Be may also act as a donor in $\mathrm{SiC}$ when residing on interstitial sites, although, the donor levels are not known. ${ }^{12}$ Despite

${ }^{a)}$ Electronic mail: sfung@hkucc.hku.hk these reports, however, the knowledge about the physics of the Be dopant in $\mathrm{SiC}$ is still very limited.

Deep level transient spectroscopy (DLTS) is one of the most direct techniques for the determination of energy states of deep level defects. Therefore, deep level traps in $p n$ junctions produced by Be implantation in $n$-type $6 \mathrm{H}-\mathrm{SiC}$ are investigated in this article by applying the DLTS technique.

\section{EXPERIMENT}

The $6 \mathrm{H}-\mathrm{SiC}$ used in this work was purchased from CREE Research Inc in the form of 10- $\mu$ m-thick nitrogen doped (0001) oriented epilayers grown on $n^{+}$type SiC substrates. The nitrogen donor concentrations were 1 $\times 10^{16} \mathrm{~cm}^{-3}$ and $8 \times 10^{17} \mathrm{~cm}^{-3}$ in the epilayer and the substrate, respectively. 50-590 keV Be implantation (termed high-dose implantation hereafter) was carried out to obtain a box-shaped profile with a mean Be concentration of about $1 \times 10^{19} \mathrm{~cm}^{-3}$. To repair the implantation induced damage and electrically activate the dopant, samples were annealed in flowing argon gas at $1873 \mathrm{~K}$ for $1 \mathrm{~min}$ using a rapid thermal annealing (RTA) system. Before metallization, about $600 \mathrm{~nm}$ was removed from the top of the epilayer by using a combination of ion implantation and wet chemical etching. ${ }^{13,14}$ Contacts were fabricated by titanium deposition on the top and nickel deposition on the backside of the samples using an electron beam evaporation system. A postdeposition RTA cycle at $1373 \mathrm{~K}$ for $5 \mathrm{~min}$ in flowing argon gas was performed to obtain ohmic contacts. Hall measurements indicated a weak $p$-type conduction in the Be implanted layer. Current-voltage measurements revealed that the as-prepared $p n$ junction switched on at a forward bias voltage of $\sim 1.5 \mathrm{~V}$, whereas a leakage current of less than 1 $\mu \mathrm{A}$ was obtained at a reverse bias voltage of $-8 \mathrm{~V}$.

For a comparative study, $6 \mathrm{H}-\mathrm{SiC}$ samples with a lower Be concentration (about $1 \times 10^{18} \mathrm{~cm}^{-3}$ ) were also prepared 
(termed low-dose implantation hereafter). The same multiple energy implantation schedule as for the high-dose implantation was applied. RTA was performed at $1873 \mathrm{~K}$ for $30 \mathrm{~s}$ under similar conditions as stated earlier. Subsequently, these samples were chemically treated in $10 \%$ hydrofluoric acid solution to remove the oxide layer, and were then rinsed in boiling acetone, ethanol, and de-ionized water. Large area ohmic contacts were made by $\mathrm{Al}$ deposition on the backside of the samples followed by an annealing process at $1173 \mathrm{~K}$ for $5 \mathrm{~min}$. Schottky contacts were prepared by depositing gold dots of $0.6 \mathrm{~mm}$ diameter on the Be implanted side of the samples. The as-prepared Schottky diodes displayed no change from $n$ - to $p$-type during the DLTS measurements conducted in the temperature range 100-450 K.

The DLTS system used in the present work has been described elsewhere. ${ }^{15}$ The measurements were carried out by applying a reverse bias of $V_{r}=-6 \mathrm{~V}$ with a forward filling pulse of $V_{p}=6 \mathrm{~V}$. Under these conditions, only majority carriers were injected into the depletion region during the filling time (electrons into the $n$ region and holes into the $p$ region of the $p n$ junction). The deep level parameters (energy levels and capture-cross sections) were determined from the slope and intercept of Arrhenius curves of thermal emission rates, whereas trap concentrations were calculated from the peak heights of the DLTS signal using the procedure described in Ref. 16. In the calculations, capture cross sections were assumed to be temperature independent. In addition, density-of-states effective masses for electrons and holes were used as in Refs. 17 and 18.

\section{RESULTS}

Recent secondary ion mass spectrometry (SIMS) investigations have revealed that the shape of the as-implanted profile changed considerably after the high temperature treatment. ${ }^{19-21}$ The reasons for this behavior were discussed elsewhere. ${ }^{20,21}$ For the high-dose implanted sample series, which is considered in the following, the Be distribution after the RTA process is characterized by a box-like profile with distinct maxima and minima as well as a long diffusion tail into the epilayer (see Fig. 1). Due to a strong in-diffusion process, this tail even reaches the underlying substrate. ${ }^{22}$ As can be further seen in Fig. 1, the Be concentration in the tail region may amount up to $6 \times 10^{16} \mathrm{~cm}^{-3}$.

A typical majority carrier spectrum is shown in Fig. 2. Five peaks labeled BE1, BE2, BE3, BE4, and BE5 were observed in the DLTS measurement temperature range 100$450 \mathrm{~K}$. To check whether these traps were induced by Be implantation, a test measurement was performed on a Schottky contacted unimplanted $n$-type epilayer. None of the earlier traps were detected indicating that all the traps described above were induced by the Be implantation process.

As stated earlier for the high-dose implanted samples, the free hole concentration in the $p$ region and the free electron concentration in the $n$ region of the $p n$ junction were of the same order of magnitude. Under reverse bias conditions it is thus reasonable to expect that the width of the depletion layer in the $p$ region is comparable to the one in the $n$ region. Consequently, the observed DLTS peaks may arise either

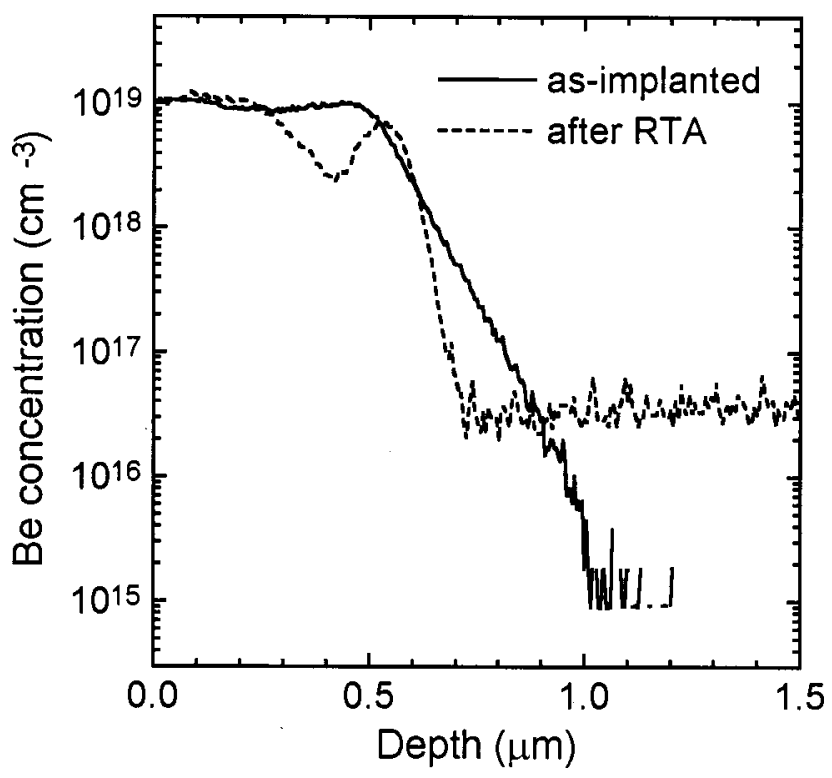

FIG. 1. Be SIMS depth profile in high-dose implanted 6H-SiC after annealing at $1873 \mathrm{~K}$. An as-implanted profile is also shown for comparison.

from hole traps in the $p$ region or electron traps in the $n$ region of the $p n$ junction. A clear assignment is not possible without further information about these traps. Therefore, the $n$-type low-dose implanted samples series was additionally prepared. A typical DLTS spectrum of these samples is shown in Fig. 3. The Be implantation generated three electron traps labeled $\mathrm{BE}^{\prime}, \mathrm{BE} 2^{\prime}$, and $\mathrm{BE} 3^{\prime}$. Since the energy level and the temperature position of the DLTS peak BE1' (see Fig. 3) are in excellent agreement with those of BE1 (see Fig. 2), BE1' and BE1 are attributed to the same defect center. Likewise $\mathrm{BE}^{\prime}$ and $\mathrm{BE} 3^{\prime}$ are assumed to be related to the overlapping peaks BE2 and BE3 because of their very similar energy levels. Hence, it is unambiguously clear that three (BE1, BE2, and BE3) of the five deep level traps ob-

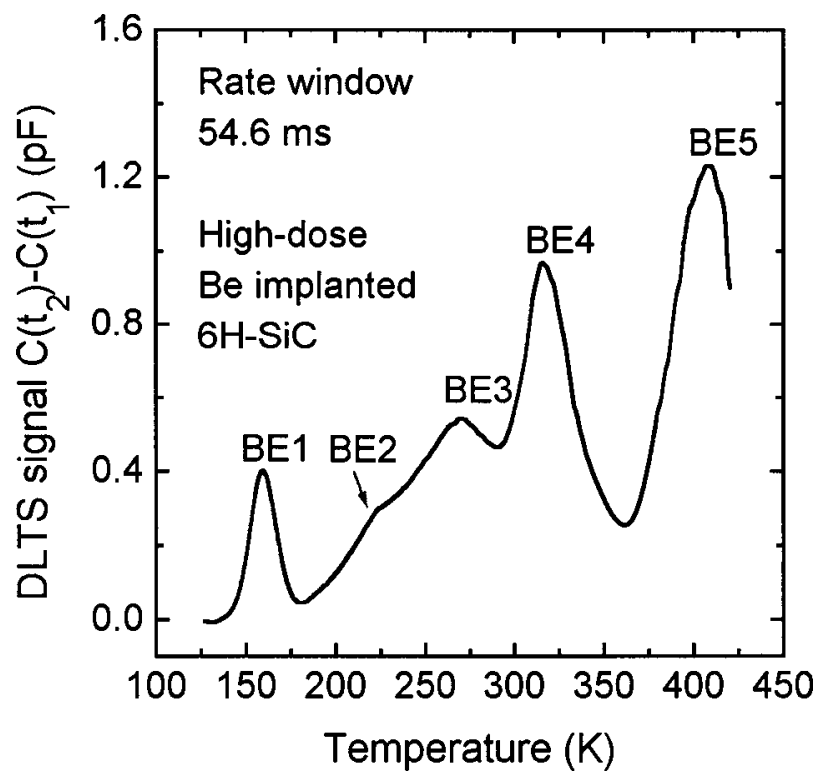

FIG. 2. DLTS majority carrier spectrum recorded on the high-dose implanted sample. 


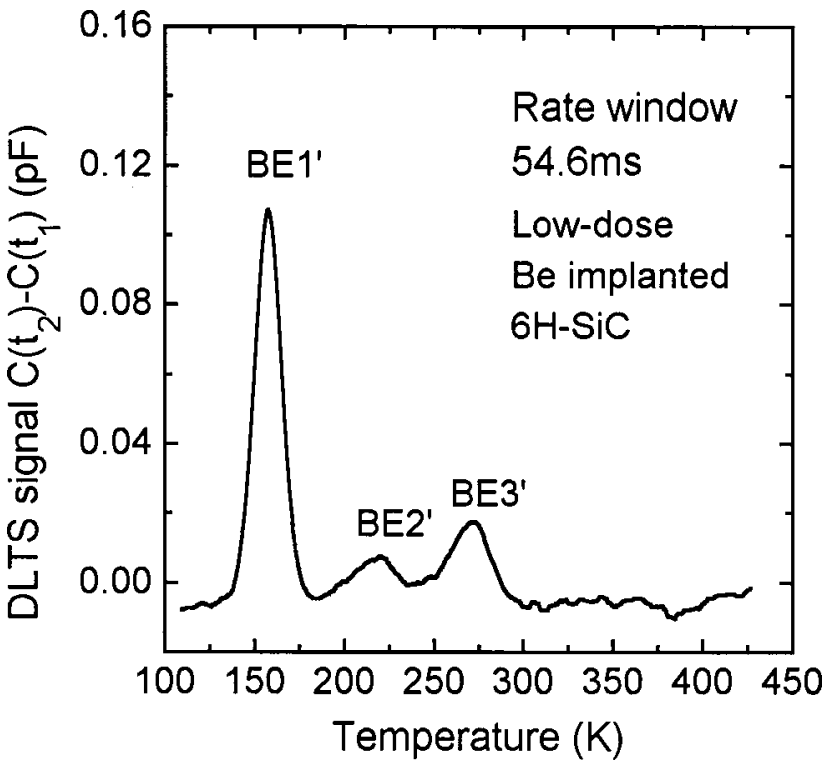

FIG. 3. DLTS majority carrier spectrum recorded on the low-dose implanted sample.

served in the high-dose implanted sample are electron traps arising from the $n$ region while the other traps (BE4, BE5) are assumed to be hole traps from the $p$ region of the $p n$ junction.

Arrhenius plots of thermal emission rates for the traps BE1-BE5 are shown in Fig. 4. Energy levels, capture-cross sections $\sigma_{T}$, and concentrations $N_{T}$ of the defect centers determined as stated earlier are summarized in Table I. In the calculations, BE1, BE2, and BE3 were assumed to be electron traps whereas BE4 and BE5 were treated as hole traps. Since the DLTS peak signals BE2 and BE3 were relatively weak and smeared, the uncertainty limits for all parameters of these traps are higher compared to the other ones.

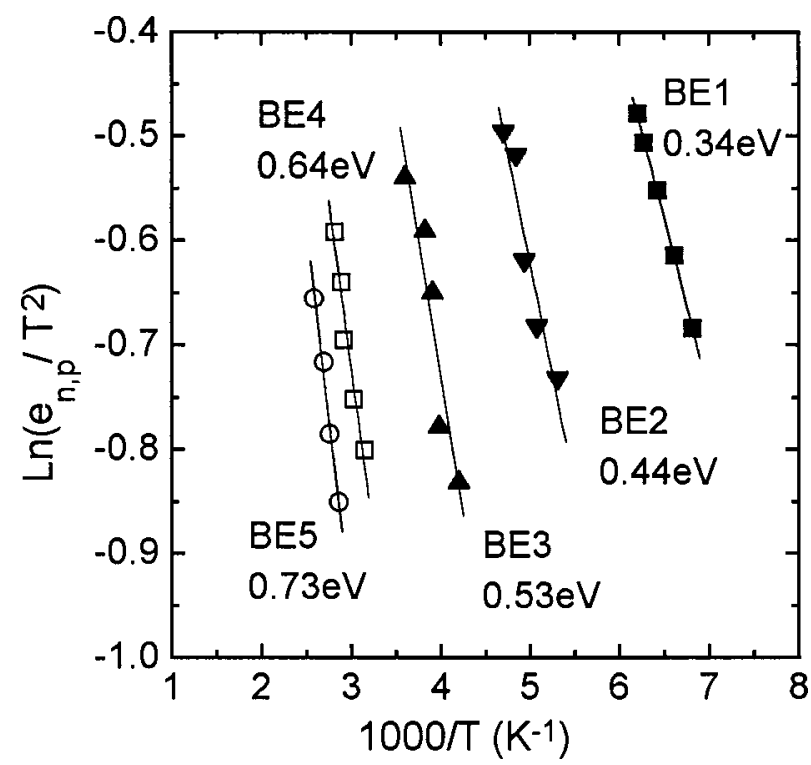

FIG. 4. Arrhenius plots of thermal emission rates for the different electron and hole traps as depicted in Fig. 2. Here $e_{n, p}$ are the emission rates for electrons and holes, respectively.
TABLE I. Energy levels $E_{T}$, capture cross sections $\sigma_{T}$, and concentrations $N_{T}$ of the deep level traps determined using DLTS data of the high-dose implanted sample.

\begin{tabular}{lccc}
\hline \hline & $E_{T}(\mathrm{eV})$ & $\sigma_{T}\left(\mathrm{~cm}^{2}\right)$ & $N_{T}\left(\mathrm{~cm}^{-3}\right)$ \\
\hline BE1 & $E_{C}-0.34 \pm 0.02$ & $\sim 10^{-13}$ & $4-10 \times 10^{13}$ \\
BE2 & $E_{C}-0.44 \pm 0.04$ & $\sim 10^{-14}$ & $1-6 \times 10^{13}$ \\
BE3 & $E_{C}-0.53 \pm 0.03$ & $\sim 10^{-14}$ & $3-8 \times 10^{13}$ \\
BE4 & $E_{V}+0.64 \pm 0.02$ & $\sim 10^{-16}$ & $4-6 \times 10^{14}$ \\
BE5 & $E_{V}+0.73 \pm 0.02$ & $\sim 10^{-16}$ & $2-7 \times 10^{14}$ \\
\hline \hline
\end{tabular}

To obtain concentration profiles of the electron traps observed in the $n$ region of the high-dose implanted $p n$ junction, further DLTS spectra were recorded using different reverse bias voltages. In the measurement procedure, the reserve bias $V_{r}$ was varied from -3 to $-8 \mathrm{~V}$, with a constant filling pulse height $\left(V_{p}=1 \mathrm{~V}\right)$ for all spectra. Figure 5 shows the normalized DLTS peak heights and thus gives some indication of the depth distribution of the defect centers. The insert shows the concentration distribution profile of BE1 evaluated from the DLTS peak heights corresponding to different reverse biases using the standard method. ${ }^{16}$ However, the distance scale is approximate as it is difficult to ascertain because we do not know accurately the effective doping in the $p$ region and the width of built-in depletion region of the Be implanted $p n$ junction. It can be seen that in contrast to peaks BE2 and BE3 which are only large at low biases (close to the $p n$ junction) the BE1 peak starts with low intensity and keeps on increasing until a reverse bias of $-6 \mathrm{~V}$ is reached. At still higher reverse biases the BE1 peak begins to reduce. This indicates that the defect responsible for the BE1

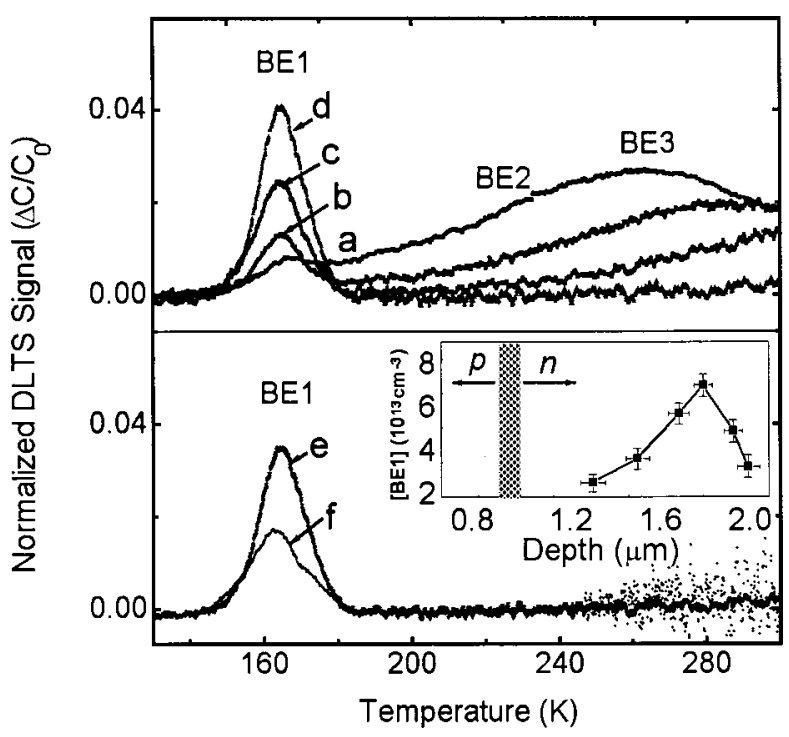

FIG. 5. Normalized DLTS spectra of the traps BE1, BE2, and BE3 recorded on the high-dose implanted sample using a filling pulse amplitude $V_{p}$ $=1 \mathrm{~V}$ on different reverse biases $V_{r}$ of (a) -3 , (b) -4 , (c) -5 , (d) -6 , (e) -7 , and (f) $-8 \mathrm{~V}$, respectively. The upper part shows the increase in peak heights of BE1 and decrease in peak heights of BE2, BE3 with increasing bias. The lower part demonstrates the decline of the peak BE1 with further increase in bias. The insert shows the concentration distribution profile of the trap BE1 evaluated from the DLTS peak heights taken at the different reverse biases. 
center becomes high in concentration at some distance from the $p n$ junction after which it begins to falloff.

\section{DISCUSSION}

\section{A. Electron traps}

The defect energy level BE1 $\left(E_{C}-0.34 \mathrm{eV}\right)$ is considered first. Recent DLTS studies on electron-irradiated ${ }^{17,22,23}$ and deuterium implanted ${ }^{22} n$-type $6 \mathrm{H}-\mathrm{SiC}$ have demonstrated the existence of two overlapping levels at $E_{C}-0.33 \mathrm{eV}$ and $E_{C}-0.34 \mathrm{eV}$ that were designated as $\mathrm{E}_{1}$ and $\mathrm{E}_{2}$, respectively. These energies may be as deep as $E_{C}-0.38 \mathrm{eV}$ and $E_{C}-0.44 \mathrm{eV}$ without correction for the thermal energy barrier of capture process. ${ }^{17}$ Both levels were attributed to one intrinsic defect. Although the microscopic structure of this $\mathrm{E}_{1} / \mathrm{E}_{2}$ center is still a matter for discussion, there is mounting evidence that it is due to the $V_{\mathrm{Si}}-V_{\mathrm{C}}$ divacancy. ${ }^{24}$

Our previous DLTS investigations on electron-irradiated $6 \mathrm{H}-\mathrm{SiC}$ revealed several deep level traps. Two of them (labeled as $\mathrm{ED}_{3}$ and $\mathrm{ED}_{4}$ ) were attributed to the $\mathrm{E}_{1} / \mathrm{E}_{2}$ center. ${ }^{23}$ The fact that the BE1 peak is so close in energy to those of the $\mathrm{E}_{1} / \mathrm{E}_{2}$ doublet is suggestive that the two are one and the same defect with the perturbations present on the center close to the $p n$ junction perhaps causing the doublet to smear into a single peak. Such a view, however, meets with some difficulty when it is realized that the peak maximum temperatures of $\mathrm{BE} 1$ and $\mathrm{ED}_{3}$ differ by $50 \mathrm{~K}$ and the calculated cross section by two orders of magnitude. This makes it unlikely that $\mathrm{BE} 1$ and $\mathrm{E}_{1} / \mathrm{E}_{2}$ are the same defect, but it does not preclude it since cross-section measurements can vary greatly in DLTS measurements based on sample preparation and the various modes of carrier scattering.

An alternative explanation for BE1 is that it is associated with interstitial $\mathrm{Be}$. It is known that Be may act as a donor when residing on interstitial positions. ${ }^{12}$ Further, there are strong evidences that Be diffuses via interstitial sites. ${ }^{12,20,21}$ Since a deep diffusion tail into the epilayer is observed after RTA (see Fig. 1) which extends even through the epilayer (not shown), there is definite evidence for fast interstitial diffusion of Be and thus by inference the related donor site. Recent $a b$ initio calculations have demonstrated that the tetrahedral lattice site with $\mathrm{C}$ neighbors is the predominant position for interstitial B. ${ }^{25}$ It may tentatively be argued by analogy that Be residing on a tetrahedral site might be the defect configuration that leads to the BE1 center. Clearly further theoretical investigations will be necessary to test this possibility.

On the other hand, it can be seen (in Fig. 5) that the BE1 center becomes high in concentration at some distance from the $p n$ junction after which it begins to falloff in the $n$ region of the high-dose $\mathrm{Be}$ implanted $p n$ junction. The displaced profile of BE1 (see Fig. 5 insert) argues against any simple interstitial Be model since the SIMS data of Fig. 1 would suggest that the Be density should be uniform in the $n$ region of the present $p n$ junction.

A strong argument can also be formulated against a simple $V_{\mathrm{Si}}-V_{\mathrm{C}}\left(\mathrm{E}_{1} / \mathrm{E}_{2}\right)$ model for $\mathrm{BE} 1$ namely that if $V_{\mathrm{Si}}-V_{\mathrm{C}}$ is formed under Be bombardment why is it not also found to do so under $\mathrm{B}, \mathrm{Al}$, and $\mathrm{Ga}$ bombardment? With these other elements there is no sign of any BE1 like energy level in the $n$ region of $\mathrm{B}, \mathrm{Al}$, and $\mathrm{Ga}$ implanted $p^{+} n$ junctions. ${ }^{6-8}$ Therefore, one possible model that concurs with the observed DLTS profile and the SIMS profile is that maybe some intrinsic damage-produced defect (such as $\left.V_{\mathrm{Si}}-V_{\mathrm{C}}\right)$ is complexing with the interstitial $\mathrm{Be}$ to form the BE1 center. Such a model would fit the observed BE1 depth profile while at the same time permitting the SIMS Be concentration to be constant in the overlayer.

Considering the peak BE2, the deep level parameters determined are close to those of the $\mathrm{ED}_{4}$ center reported previously. ${ }^{23}$ However, the peak in the DLTS spectrum is very weak and overlapped by the peak BE3, which limits the precision of the calculated parameters. Therefore, the question whether the trap BE2 is to be associated with an implantation induced intrinsic defect or a Be induced defect cannot be answered at present.

The trap BE3, the energy level of which has been determined as $E_{C}-0.53 \mathrm{eV}$, is similar to the defect level was previously found in electron irradiated $6 \mathrm{H}-\mathrm{SiC}$ by several groups. ${ }^{17,22,23}$ Investigations with respect to the thermal stability of this defect center revealed, however, that this trap anneals out at around $573 \mathrm{~K}^{22,23}$ Hence, the $\mathrm{C}$ vacancy, which is known to represent a similar annealing behavior, was suggested as a possible model for this intrinsic defect. ${ }^{22}$

On the other hand, Dalibor $e t$ al. observed a trap labeled $\mathrm{ID}_{7}$ at $E_{C}-0.50 \mathrm{eV}$ after vanadium as well as titanium implantation followed by an annealing process at $1973 \mathrm{~K}^{26}$ This defect level was ascribed to an implantation induced intrinsic defect center. Due to the different annealing behavior, the defect associated with the $\mathrm{ID}_{7}$ center must be different from the one observed in electron irradiated material. Further, the capture-cross sections determined for the $\mathrm{ID}_{7}$ and the BE3 center are in the same order of magnitude. The BE3 center, however, was not detected by DLTS in our unimplanted virgin material. Therefore, we believe that both levels originate from the same defect, i.e., an intrinsic defect center induced by the implantation process.

\section{B. Hole traps}

Substitutional Be introduces two acceptor states within the band gap that were observed by Hall measurements. ${ }^{10}$ However, it is unlikely that the first of the two charge states can be detected in our samples by DLTS. The Fermi level in the $p$ region of the $p n$ junction is expected to be above the first acceptor level. Thus, under reverse bias conditions, the first acceptor level is always filled with electrons during the filling pulse. Since hole traps associated with intrinsic defects having a similar energy level as BE4 have not been observed so far, the BE4 center located at $E_{V}+0.64 \mathrm{eV}$ is assumed to originate from the second charge state of the Be acceptor. The different measurement techniques and purities of the samples are assumed to be responsible for the small discrepancy between the DLTS and the Hall result $\left(E_{V}\right.$ $+0.6 \mathrm{eV}$ ) reported in the past. ${ }^{10}$

The deepest defect level BE5 has been characterized as a hole trap at $E_{V}+0.73 \mathrm{eV}$. Though comprehensive investigations on intrinsic defects in $p$-type $6 \mathrm{H}-\mathrm{SiC}$ are still lacking, a 
defect level labeled $\mathrm{H} 2$ at a similar energy position was found in electron irradiated material by our group. ${ }^{27}$ It was demonstrated that the defect associated with $\mathrm{H} 2$ anneals out at about $600 \mathrm{~K}$. Therefore, it is rather unlikely that BE5 and $\mathrm{H} 2$ originate from the same defect. At present we believe that the BE5 center can be associated with a Be induced complex.

Very recently, several deep Be acceptor centers were found by electron paramagnetic resonance and electronnuclear double resonance investigations on $6 \mathrm{H}-\mathrm{SiC}$ crystals doped by diffusion technique. ${ }^{28}$ These defect centers were assigned to the $E_{V}+0.6 \mathrm{eV}$ level determined by Hall measurements. ${ }^{10}$ However, this association is questionable since a complex defect consisting of an acceptor and an intrinsic defect (e.g., A vacancy) generates a level within the band gap which is usually deeper than the one related to a simple substitutional acceptor. Therefore, we believe that the BE5 center is to be associated with these defect centers which were found to resemble the deep B center in terms of electronic and magnetic properties. ${ }^{29}$ Though the exact defect configuration is still under debate, a complex consisting of a $\mathrm{B}$ atom residing on a $\mathrm{Si}$ site and an adjacent $\mathrm{C}$ vacancy seems to be the most favored model for the deep B center. ${ }^{29}$ In analogy, a complex consisting of a substitutional Be atom and a nearest-neighbor vacancy is therefore suggested as defect model for the BE5 center. Further experimental and theoretical investigations are necessary to clarify the microscopic structure of BE5 and the other Be implantation induced defects.

\section{CONCLUSIONS}

Deep level traps induced by Be implantation in $6 \mathrm{H}-\mathrm{SiC}$ were investigated by DLTS in the temperature range 100450 K. Five deep level traps labeled BE1, BE2, BE3, BE4, and BE5 were detected in $p n$ junctions produced by Be implantation.

The BE1, BE2, and BE3 centers were found to be electron traps located at $0.34,0.44$, and $0.53 \mathrm{eV}$, respectively, below the conduction band edge. The identity of BE1 center was discussed as an associated interstitial Be defect, whereas the BE3 center was attributed to an implantation induced intrinsic defect. On the other hand, the BE4 and BE5 centers were found to be hole traps which are situated at 0.64 and $0.73 \mathrm{eV}$, respectively, above the valence band edge. The BE4 center was attributed to the second charge state of the $\mathrm{Be}$ acceptor, whereas the BE5 center is believed to originate from a Be induced complex in SiC.

\section{ACKNOWLEDGMENTS}

This work was partially supported by grants from the Research Grant Council of the Hong Kong Special Admin- istrative Region, China (under Project Nos. HKU7101/97P and HKU7137/99P). S. Fung also acknowledges partial support from the HKU CRCG grant.

${ }^{1}$ G. L. Harris, Properties of Silicon Carbide (INSPEC, London, 1995), p. 153.

${ }^{2}$ Y. A. Vodakov and E. N. Mokhov, Silicon Carbide-1973 (University South Carolina Press, Columbia, 1974), p. 508.

${ }^{3}$ W. Suttrop, G. Pensl, and P. M. Laning, Appl. Phys. A: Solids Surf. 51, 231 (1990).

${ }^{4}$ M. V. Rao, P. Griffiths, O. W. Holland, G. Kelner, J. A. Freitas, Jr., D. S. Simons, P. H. Chi, and M. Ghezzo, J. Appl. Phys. 77, 2479 (1995).

${ }^{5}$ T. Troffer, M. Schada, T. Frank, H. Itoh, G. Pensl, J. Heindl, H. P. Strunk, and M. Maier, Phys. Status Solidi A 162, 277 (1997).

${ }^{6}$ M. Gong, C. V. Reddy, C. D. Beling, and S. Fung, Appl. Phys. Lett. 72, 2739 (1998).

${ }^{7}$ S. Fung, M. Gong, and C. D. Beling, J. Appl. Phys. 84, 1152 (1998).

${ }^{8}$ M. Gong, S. Fung, and C. D. Beling, J. Appl. Phys. 85, 105 (1999).

${ }^{9}$ T. Troffer, G. Pensl, A. Schöner, A. Henry, C. Hallin, O. Kordina, and E. Janzen, Mater. Sci. Forum 264-268, 557 (1998).

${ }^{10}$ Y. P. Maslakovets, E. N. Mokhov, Y. A. Vodakov, and G. A. Lomakina, Sov. Phys. Solid State 10, 634 (1968).

${ }^{11}$ N. Ramungul, Y. P. Zheng, R. Rupal, and T. P. Chow, IEEE Trans. Electron Devices 46, 465 (1999).

${ }^{12}$ Y. A. Vodakov, G. A. Lomakina, E. N. Mokhov, V. G. Oding, and E. I. Radovanova, Sov. Phys. Solid State 20, 258 (1978).

${ }^{13}$ D. Alok and B. J. Baliga, J. Electron. Mater. 24, 311 (1995).

${ }^{14}$ T. Henkel, G. Ferro, S. Nishizawa, H. Pressler, Y. Tanaka, H. Tanoue, and N. Kobayashi, Proceedings of the 11th International Conference on Silicon Carbide and Related Materials (ICSCRM'99), North Carolina, 1999.

${ }^{15}$ C. V. Reddy, S. Fung, and C. D. Beling, Rev. Sci. Instrum. 67, 257 (1996).

${ }^{16}$ D. V. Lang, J. Appl. Phys. 45, 3023 (1974).

${ }^{17}$ C. Hemmingsson, N. T. Son, O. Kordina, and E. Janzen, J. Appl. Phys. 84, 704 (1998).

${ }^{18}$ G. Wellenhofer and U. Rössler, Phys. Status Solidi B 202, 107 (1997).

${ }^{19}$ T. Henkel, Y. Tanaka, N. Kobayashi, H. Tanoue, M. Gong, X. D. Chen, S. Fung, and C. D. Beling, Proceedings of MRS 1999 Spring Meeting (San Francisco, CA, 1999), MRS 572, 117.

${ }^{20}$ T. Henkel, Y. Tanaka, N. Kobayashi, S. Nishizawa, and S. Hishita, Proceedings of the 11th International Conference on Silicon Carbide and Related Materials (ICSCRM'99), North Carolina, 1999.

${ }^{21}$ T. Henkel, Y. Tanaka, N. Kobayashi, H. Tanoue, and S. Hishita, Appl. Phys. Lett. (submitted).

${ }^{22}$ M. O. Aboelfotoh and J. P. Doyle, Phys. Rev. B 59, 10823 (1999).

${ }^{23}$ M. Gong, S. Fung, C. D. Beling, and Z. You, J. Appl. Phys. 85, 7604 (1999).

${ }^{24}$ T. Frank G. Pensl, S. Bai, R. P. Devaty, and W. J. Choyke, Proceedings of the 11th International Conference on Silicon Carbide and Related Materials (ICSCRM'99), North Carolina, 1999.

${ }^{25} \mathrm{M}$. Bockstedte and O. Pankratov, Proceedings of the 11th International Conference on Silicon Carbide and Related Materials (ICSCRM'99), North Carolina, 1999.

${ }^{26}$ T. Dalibor, G. Pensl, H. Matsunami, T. Kimoto, W. J. Choyke, A. Schoener, and N. Nordell, Phys. Status Solidi A 162, 199 (1997).

${ }^{27}$ M. Gong, S. Fung, C. D. Beling, and Z. You, J. Appl. Phys. 85, 7120 (1999)

${ }^{28}$ A. van Duijn-Arnold, J. Schmidt, O. G. Poluektov, P. G. Baranov, and E. N. Mokhov, Phys. Rev. B 60, 15799 (1999).

${ }^{29}$ A. van Duijn-Arnold, T. Ikoma, O. G. Poluektov, P. G. Baranov, and J. Schmidt, Phys. Rev. B 57, 1607 (1998). 